\title{
DETERMINANTES DE \\ CLASE EN EL SISTEMA \\ EDUCATIVO
}

\section{Rafael Ávila Penagos*}

\section{EL PUNTO DE PARTIDA}

Circulan por los pasillos académicos toda una serie de postulados que muy pocos se preocupan por verificar o por falsificar: el mito de la movilidad social por la vía de la educación, la hipótesis de que una desigual distribución de oportunidades educativas causa deferencias de clase; y la estrategia, montada sobre dicha hipótesis, según la cual una redistribución de oportunidades educativas o la simple expansión del cubrimiento educativo, puede remover, al menos, las enormes desigualdades sociales que afectan a nuestra sociedad.

El mérito académico del profesor Gonzalo Cataño consiste, a mi parecer, en poner en jaque dichos postulados e hipótesis. El, apartándose de las opiniones circulantes, sostiene todo lo contrario: es la estructura de clases la que determina un acceso diferencial a la educación; y es esa misma estructura la que produce una diferenciación interna del sistema educativo, según rangos de escolaridad.

Su argumentación puede encontrarse básicamente en dos artículos suyos, ambos publicados en la Revista Colombiana de Educación: el primero, cuando esta Revista hacia apenas su presentación en sociedad, intitulada "Educación y clase social en Colombia"t (1975); y el segundo, nueve años más tarde (1984), en el No. 14 de la misma, bajo el título de "Educación y diferenciación social en Colombia".

Más afortunado el título del primero que el del segundo, este resultó más enjundioso que aquel. La preocupación por la misma problemática se mantiene, pero en el segundo perfecciona su batería teórica, eleva su nivel argumentativo, y desarrolla matizando algunos cabos que le habían quedado sueltos en el primero.

Un análisis somero y algunos comentarios a estos dos artículos constituyen el objetivo de este ensayo.

\section{SOBRE EL CONCEPTO DE CLASE}

Sí en $\mathrm{e}^{1}$ primer artículo el autor utiliza, sin definir, el concepto de clase social, asociado a la imagen bien vaga de estrato, el segundo artículo aporta una definición de clase montada sobre cuatro características:

- Agregados de individuos y familias con posiciones socioeconómicas similares.

\footnotetext{
* Doctor en Sociología Univ.Católica de Lovaina

Prof. Educación y Sociedad. Univer. Pedagógica Nacional 
- Con diferentes capacidades de acceso a bienes y servicios.

- Según su propiedad, sus ingresos y su ocupación.

- Con un modo de vida típico que permite identificarlas como "subculturas".

Nos parece interesante en esta definición señalar el aspecto cultural de una clase social, pero creemos que en ella no están lo suficientemente caracterizados tres elementos relevantes para la tradición dialéctica:

Su relación con los medios de producción, su papel en la organización social del trabajo y su grado de participación en el producto social global.

La sociología accionalista por su parte, propone tener en cuenta otro parámetro: el control de la historicidad, o la capacidad que una sociedad tiene de actuar sobre sí misma. El concepto de historicidad (Touraine) no dista mucho, a nuestro juicio del concepto de hegemonía (Gramsci).

\section{CLASES SOCIALES Y ACCESO AL SISTEMA EDUCATIVO}

Para sustentar la influencia de la estructura de clases en la diferencia de acceso al sistema educativo, estos son sus principales argumentos:

1. Mientras que, en la ciudad, los sectores "populares" se escolarizan en "escuelas", los sectores medios y altos se escolarizan en "colegios".

2. Mientras que, en el campo, aparceros, arrendatarios y peones escolarizan a sus hijos en las escuelas rurales; los latifundistas, medianos propietarios y mini empresarios escolarizan a sus hijos en los colegios de provincia de mayor rango.

3. Mientras que artesanos, pequeños propietarios, pequeños comerciantes, y funcionarios públicos de poblaciones intermedias escolarizan a sus hijos en institutos técnicos, agrícolas, de comercio o en normales; los hijos de la élite política y económica escolarizan a sus hijos en las Universidades de mayor prestigio.

4. Más aún: la estructura de clases se expresa también en una jerarquía de status entre las instituciones, determinadas por el rango de las profesiones. Rango que, a su vez, está determinado por la valoración que los sectores de clase dominantes hacen del conjunto de saberes más relacionados con su situación de poder.

Para mantener el poder hay que valorar más el saber del poder que el poder del saber.

5. Para el caso de toda la población docente, su prestigio tiene una unidad de medida: el origen social de su audiencia estudiantil.

\section{CLASES SOCIALES Y DINAMICA INTERNA DEL SISTEMA EDUCATIVO}

Para sustentar la influencia de la estructura de clases sobre la dinámica interna del sistema educativo, recurre a los siguientes argumentos: 
1. Cada sector de clase tiende a escolarizar a sus hijos en instituciones diferentes. Queda aquí subentendido que esto es posible cuando, y sólo cuando, el sistema educativo ha alcanzado un cierto nivel de complejidad que le permite diferenciar en instituciones para las masas e instituciones para las élites, en cualquiera de los niveles de la educación, o al menos en algunos.

2. Una conclusión diferente resulta de la contrastación de la teoría con un nuevo material empírico (el caso de Aritama).

"En las sociedades con marcadas diferencias de clase y con sistemas educativos muy restringidos, esto es, donde no hay lugar para instituciones populares y de élite, las divisiones sociales se introducen en el salón de clase".

En otras palabras: Hay situaciones en donde los diferentes sectores de clase se ven obligados a escolarizar a sus hijos en la misma institución. Caso en el cual las diferencias de clase, obviamente, se trasladan al interior de esa institución.

3. Habría que introducir aquí una variante para la reflexión.

a. Cuando la existencia de una sola institución es debida a la baja complejidad del sistema educativo y a su subdesarrollo (en una sociedad subdesarrollada).

b. Cuando la existencia de una sola institución es el resultado de una decisión política del Estado.

\section{UNA HIPOTESIS INSPIRADA EN BOURDIEN}

Aunque el profesor Cataño visualiza y plantea el problema con el instrumental categorial propio de la tradición parsoniana -procedimiento absolutamente legitimo- nos parece de utilidad ampliar su argumentación con una hipótesis de inspiración bourdieuana que apenas si es tratada de soslayo en su trabajo. Me refiero a la diferenciación jurídico-legal del sistema educativo en una rama pública y una rama privada.

Para introducirnos conviene recordar que la forma de organización del Estado está determinada por la lógica económica que subyace al ordenamiento jurídico, según que el sector público de la economía esté supeditado al sector privado (cuando el régimen de acumulación y su modo de regulación están bajo el control de un bloque hegemónico conducido por la burguesía) o, el caso contrario, cuando el sector privado de la economía está supeditado al sector público (caso en el cual el bloque económico que conduce la reproducción del régimen de acumulación y su modo de regulación está conducido por sectores diferentes a la burguesía).

Esta misma lógica económica rige también el ordenamiento jurídico-legal del sistema educativo, la cual conduce a diferenciar su estructura interna en una doble forma de institucionalización que, en el vocabulario jurídico-legal, es consagrada como la rama pública y la rama privada del sistema educativo colombiano, con funciones diferentes pero complementarias.

Atribuyendo, como le atribuimos, a esta tesis una inevitable pertinencia científica y política, tratamos a continuación de expresarla en forma de hipótesis, señalando a continuación cuáles serían las razones que permiten conducir a su verificación. 
Dicha hipótesis puede formularse como sigue:

La reproducción (educativa) de las relaciones sociales de producción (de tipo capitalista) se operacionaliza por la vía de un régimen de control sobre dos tipos de procesos:

a. El acceso a los medios de producción de la cultura.

b. La distribución del patrimonio cultural.

Este régimen de control se materializa en una doble forma de institucionalización que bifurca el acceso al sistema educativo en dos ramas: la pública y la privada.

Las pistas que permiten conducir a la verificación de esta hipótesis pueden ser las siguientes:

\subsection{Las funciones de la educación pública}

1. Promueven una estrategia de respuesta a la demanda creciente de sectores populares, centrada en aspectos principalmente cuantitativos como: la expansión rápida del sistema, la ampliación de la cobertura, el enganche de agentes socializadores con niveles bajos de calificación, la ampliación de espacios, de jornadas, y de población-meta, etc.

2. Promueven una estrategia de selección para "el ejercicio de actividades auxiliares e instrumentales" (Decreto 80 de 1980, art.26), a las cuales podemos llamar oficios, aunque el decreto las llama "educación vocacional".

3. Promueven una estrategia de selección de los saberes, pero principalmente de los saber-hacer requeridos por dichas actividades auxiliares. Es decir funcionan como un canal que distribuye los saberes estrictamente necesarios para el entrenamiento en dichos oficios.

4. La normatividad oficial para este sector es más voluminosa y más taxativa.

\subsection{Las funciones de la educación privada}

1. Promueven una estrategia de respuesta a los sectores de clase media y alta que tienden a privilegiar aspectos cualitativos como: mejoramiento del currículo, control de cobertura, enganche de agentes socializadores con mayores niveles de calificación, cualificación de espacios, disminución de jornadas y de población-meta.

2. Promueven una estrategia de selección para las profesiones más estrechamente relacionadas con el saber del poder (El Decreto 80 de 1980 califica a esta preparación de "educación superior").

3. Promueven una estrategia de selección de los saberes requeridos para el ejercicio de dichas profesiones, favoreciendo las condiciones de acumulación y desarrollo del capital cultural propio de estos sectores de clase.

4. La normatividad oficial de este sector es más flexible y más diferenciada.

Esta doble forma de institucionalizar el acceso al sistema educativo está pues marcada por su impronta de clase. Al bifurcar el acceso al patrimonio cultural, se produce un acceso disimétrico a los medios de producción de la cultura; lo cual implica un acceso disimétrico al mercado laboral y, por consiguiente, a la división social del trabajo en el 
sistema de producción. La distribución disimétrica de la cultura queda materializada en el contraste entre la calificación requerida para los oficios, y la calificación requerida para las profesiones, ocupando entre estas últimas un lugar bien privilegiado, aquellas que mas se relacionan con el saber del poder (léase el saber requerido por el bloque hegemónico para mantener su poder).

\section{LA ASIMETRIA DEL MERCADO LABORAL}

El Estado colombiano ha sabido inventar variantes originales de la educación pública mediante acuerdos con el capital privado (EL SENA por ejemplo) para calificar recursos humanos que según los expertos de la OIT "han podido satisfacer las necesidades tanto cuantitativas como cualitativas de mano de obra industrial, en especial mano de obra a nivel intermedio" (OIT, Hacia el pleno empleo, citado por Ivon Lebot, DANE 1978, pg. 139).

Estas y otras variantes (ITAS e INEM) muestran que el Estado colombiano ha sabido crear las condiciones para propiciar la mayor oferta posible de fuerza laboral calificada.

Como el antiguo negociante de esclavos, el Estado ha constituido toda una red institucional para captar población proveniente de los estratos más bajos a fin de producir esclavos más o menos calificados que puedan engrosar el mercado laboral.

Esta institución es precisamente la que necesitan los empleadores para poder comprar una fuerza laboral calificada, seleccionada y lista para ser empleada, al precio que el empleador impone. La relación asimétrica entre compradores y vendedores de fuerza de trabajo es la que determina la valoración (el precio) asignado a su trabajo.

Hoy, a diferencia de antaño, en lugar de mirarle los dientes, se le examinan sus credenciales académicos. Según su tipo y nivel puede haber garantía de mayor o menor productividad.

Entre más calificado está el esclavo tiene mayores oportunidades de ser comprado, pero ni el nivel ni el tipo de calificación son garantía para ser enganchado. De esta manera nos encontramos ante una arbitrariedad: los empleadores le fijan al sistema educativo la finalidad de aumentar los niveles de calificación, de productividad y de eficacia, determinan los prerrequisitos laborales y exigen una calificación específica para cada ocupación. Sin embargo, a la postre, se reservan el derecho de modificar los requerimientos para el empleo, cuando la presión de la oferta laboral los acucia.

Esto prueba, una vez más, que las relaciones entre educación y empleo son más de naturaleza política que de naturaleza tecnoeconómica; y que están más determinadas por la asimetría de los mercados de trabajo que por la supuesta adecuación entre calificación de la fuerza laboral (oferta) y los requisitos para el empleo (demanda). La asimetría del mercado laboral, expresa entonces la asimetría característica de las relaciones sociales de producción capitalista, bajo su doble modalidad de relación mercantil y relación salarial.

\section{SOBRE EL PROBLEMA DE LA MOVILIDAD SOCIAL}

Hemos dicho ya que un acceso diferencial al sistema educativo implica una distribución disimétrica y sesgada del patrimonio cultural que pone en evidente desigualdad a unas 
clases, pre-destinadas a los oficios, con respecto a otras: las pre-destinadas a las profesiones.

Estas premisas, sin embargo, no constituyen argumentación alguna para invalidar la tesis de la movilidad por la vía de la educación. Para mostrar que esta invalidación está lejos de ser evidente proponemos considerar los siguientes argumentos:

7.1. En sociedades pertenecientes a la constelación del capitalismo dependiente, como es la nuestra, los diferenciales de ingreso entre los diferentes niveles de la educación son proporcionalmente altos, mientras que en las sociedades pertenecientes al capitalismo de los centros, los diferenciales de ingreso son proporcionalmente bajos.

La anterior proposición no es otra cosa que el resultado de una lectura cuidadosa del siguiente cuadro:

CUADRO No. 26

\section{RELACION PORCENTUAL ENTRE INGRESOS Y NIVEL EDUCATIVO EN COLOMBIA Y OTROS PAISES**}

\begin{tabular}{lclclr}
\hline & $\begin{array}{l}\text { PRIMARIA } \\
\text { SUPERIOR }\end{array}$ & $\begin{array}{l}\text { SECUNDARIA } \\
\text { SUPERIOR }\end{array}$ & & \multicolumn{2}{l}{ SUPERIOR } \\
\hline Países & Ninguno & Primaria & Secundaria & Primaria & Ninguno \\
\hline Colombia & 300 & 300 & 150 & 450 & 1.350 \\
Chile & 167 & 222 & 200 & 488 & 815 \\
México & 332 & 140 & 225 & 315 & 1.046 \\
Noruega & -- & 140 & 150 & 213 & -- \\
EE.UU. & -- & 148 & 165 & 244 & -- \\
Malasia & 381 & 428 & 180 & 773 & 2.949 \\
\hline
\end{tabular}

Fuente: SENA, Educación, formación profesional y empleo, Bogotá, Mayo de 1984, pg. 90.

Salta a la vista que en el país más subdesarrollado de la muestra se constatan los mayores diferenciales de ingreso entre los salarios por nivel educativo.

En países como Noruega y EE.UU. donde la mayoría de la población ha terminado el nivel primario, y donde las condiciones de vida son mejores, la diferencia de ingresos entre los diferentes niveles de educación es proporcionalmente baja.

Mientras que en países como Colombia y México donde un buen porcentaje de la población no ha accedido a la educación primaria, y donde sus condiciones de vida son inferiores, la diferencia de ingresos según niveles de educación es relativamente alto. Malasia es el caso extremo.

A medida que ascendemos en el nivel de escolaridad los rendimientos en términos de ingresos son crecientes. Esto es válido para todos los países de la muestra. Lo que varía es la proporción según el nivel de desarrollo.

\footnotetext{
${ }^{* *}$ Para la lectura de este cuadro debe tenerse en cuenta que los números indican la relación porcentual, mientras que las columnas indican los niveles educativos en comparación
} 
Pero cuidado, aunque este cuadro parece llevarnos a la conclusión de que, en nuestros países, la escolaridad es más rentable que en los países desarrollados, lo es solamente para una minoría de la población que ha logrado acceder a los niveles más altos de la pirámide educativa. Mientras que la rentabilidad de la escolaridad en los países desarrollados es proporcionalmente menor debido a la ausencia de analfabetismo y a la mejor distribución de su población en los diferentes niveles de la educación.

Diferenciales de ingreso proporcionalmente menores reducen las diferencias de clase en los países desarrollados, mientras que diferenciales de ingreso proporcionalmente altos amplían esas diferencias en los países del capitalismo dependiente.

Esta situación es la que, a mi juicio, permite alimentar la ilusión (léase bien: la ilusión) de que los profesionales e intelectuales mejor remunerados se asimilan a las clases dominantes, cuando en realidad es sólo eso: un efecto de asimilación ilusoria que los sitúa por encima de los más, pero que nunca los hace cambiar de clase en sentido estricto. Movilidad entones sí la hay, pero sólo para las minorías, y a un costo político enorme: poner a rentar su capital cultural a favor de la clase dominante. El resultado final: la subordinación de la inteligencia de una clase a los intereses de la otra.

7.2. Los diferenciales de ingreso han disminuido pero permanecen. Es lo que muestran $B$. Kugler y Martha 1. de Gómez en una tabla comparativa contrastando los diferenciales de 1975 con los de 1980. Ellos no explican esta reducción, y aunque nosotros podríamos formular la hipótesis de su relación con la ampliación de cobertura, más nos interesa para los efectos de este ensayo señalar que la correlación entre nivel de escolaridad e ingresos queda en pie.

CUADRO No. 7

DIFERENCIALES DE INGRESO ENTRE LOS SALARIOS REALES MEDIOS POR NIVEL EDUCATIVO 1975-1980

\begin{tabular}{|l|c|c|c|c|}
\hline & \multicolumn{2}{|c|}{1975} & \multicolumn{2}{c|}{1980} \\
\hline & Primaria & Secundaria & Primaria & Secundaria \\
\hline Primaria & & & & \\
\hline Secundaria & $+63 \%$ & & & $+36 \%$ \\
\hline Superior & $+350 \%$ & $+175 \%$ & $+202 \%$ & $+123 \%$ \\
\hline
\end{tabular}

Fuente: Kugler y de Gómez, cuadro No. 17.

7.3. Un mayor nivel de escolaridad determina una mejor capacidad para asimilar una experiencia de trabajo en menor tiempo, capacidad que termina siendo reconocida por una elevación más rápida en su nivel de salarios.

Es lo que podemos deducir de una lectura detenida de la siguiente gráfica 


\section{Figura 1 \\ CAMBIOS EN EL INGRESO MENSUAL, DERIVADOS DE AÑOS ADICIONALES DE EXPERIENCIA SEGUN NIVELES EDUCATIVOS}

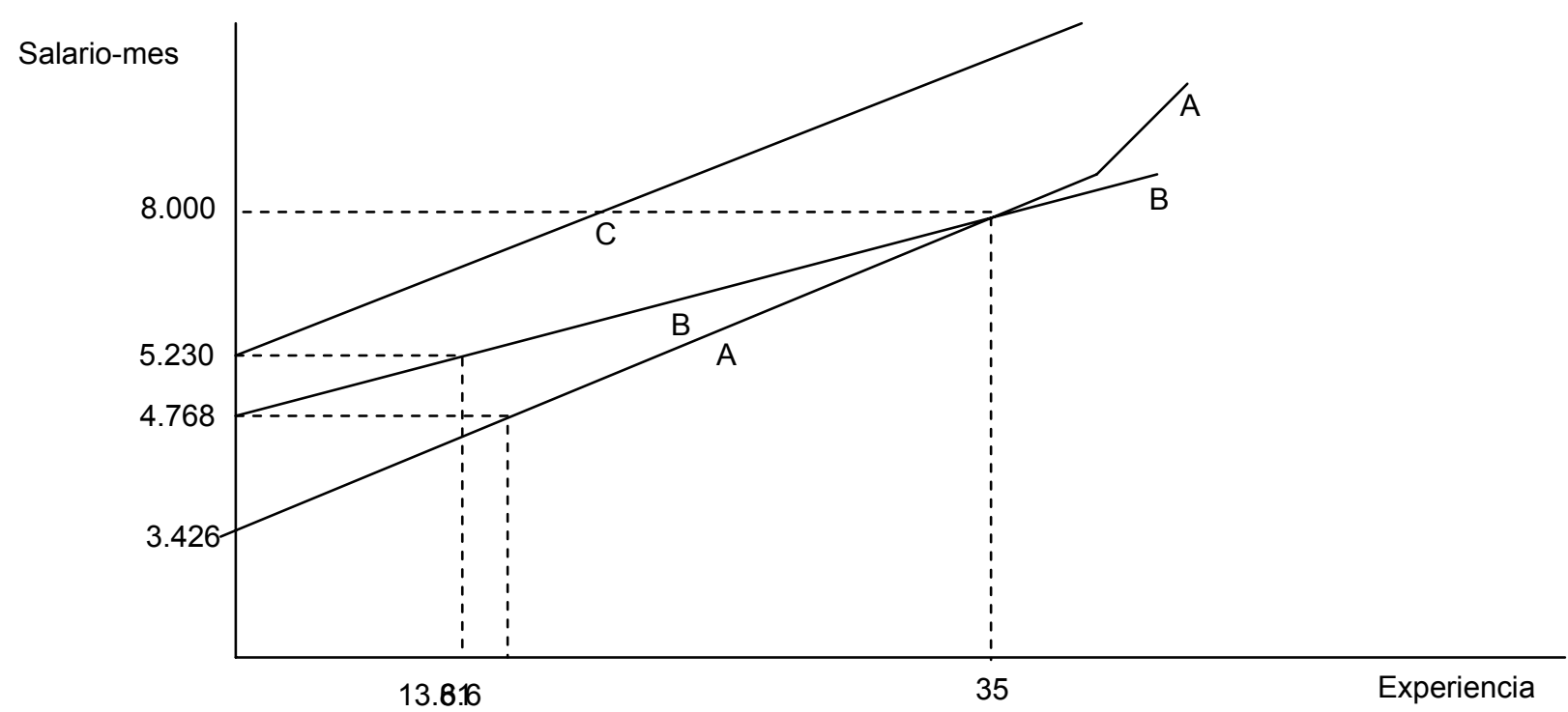

Fuente: Reu. Desorrollo y Sociedod, UNIANDES, No. 7,1982, pág. 153

A = Variación de salarios para trabajadores sin alguna educación.

$B=$ Variación de salarios de los trabajadores que han cursado por lo menos un año de primaria.

$C=$ Variación de salarios de los trabajadores que tienen algún año de secundaria.

La trayectoria de A nos muestra cómo los operarios sin ninguna educación, además de comenzar con un salario muy bajo, se tardan casi 14 años para alcanzar el nivel inicial de ingresos de aquellos que han pasado por la educación primaria.

Sólo a un plazo muy largo, 35 años, las diferencias de ingresos de los dos grupos vienen a emparejarse.

La trayectoria de B alcanza en un período más corto el nivel inicial de salarios de los trabajadores representados por $\mathrm{C}$, pero a largo plazo nunca logran alcanzar su nivel de salarios.

En cuanto a la trayectoria de $\mathrm{C}$ la curva muestra claramente tanto la diferencia de salario en el punto de partida, como la tendencia a mantener y acentuar esa diferencia en un tiempo proporcionalmente más corto. En 15 años logran los niveles salariales que A y B tardaron 35 años en alcanzar.

Queda pues claro que el logro de un nivel salarial más alto en un tiempo relativamente corto, está determinado por un nivel más alto de escolaridad en el punto de partida. 
Si el cambio en el nivel de salarios es uno de los indicadores válidos para identificar un cambio de status, tenemos aquí un nuevo argumento para verificar el postulado de la movilidad por la vía de la educación.

Claro que la movilidad a la cual nos referimos aquí es válida para $\mathrm{N}$ número de individuos, difícilmente cuantificable, y no para "un movimiento masivo de individuos y grupos de un estrato social a otro" (Cataño 84, pg. 49).

\section{EL CONTRASTE ENTRE DOS PERSPECTIVAS}

La categoría de movilidad en la tradición parsoniana visualiza al actor como unidad de análisis, y no a un "movimiento social".

La tradición parsoniana, más preocupada por el actor que por la clase social se inclina más a seguir la trayectoria del actor en una especie de continuum (ascendente 0 descendente) que a seguir la trayectoria conflictual y contradictoria de la clase en un campo de fuerzas, tal como lo hace la tradición marxista.

Dos maneras de conceptualizar el movimiento están en juego; la tradición parsoniana propone medir el movimiento de un actor social a través de indicadores tales como el nivel de escolaridad o el nivel de ingresos.

La tradición marxista en cambio recurre a otra unidad de medida: el actor se mueve cuando la clase social a la cual pertenece modifica a su favor su posición en un campo de fuerzas sociales.

Según la primera tradición el conocimiento de un actor puede ser teorizado como capital para negociar su posición en una escala jerárquica, pero es valorado únicamente por su rentabilidad en el sistema de producción. Para la segunda tradición los saberes, los valores y las costumbres de una clase social también pueden ser visualizados como "capital cultural" pero su rentabilidad no es medida por su capacidad de acople con el sistema de producción, sino por su capacidad para reforzar o debilitar la posición de esa clase social en un campo de fuerzas. La primera está más atenta al desarrollo cognitivo del actor, la segunda a la génesis y a las formas de la conciencia social.

Estamos pues ante dos conceptualizaciones del movimiento social y dos formas de autocomprender el conocimiento que pueden no excluirse mutuamente a condición de recibir un tratamiento adecuado, pero lo que más nos preocupa es que pueden también inspirar proyectos pedagógicos de signos bien diferentes con centros de perspectiva diferentes. 


\section{BIBLIOGRAFIA}

BOURDIEU, P. y J. C. Passeron La reproduction, éléments pour une théorie du système d'enseignement. Ed. Minuit, Paris, 1970.

CATAÑO, Gonzalo. Educación y clase social, en Revista Colombiana de Educación No. 1.

Educación y Diferenciación social en Colombia, en Revista Colombiana de Educación, No.14, págs. 39-51.

DANE Estudio de lijan Lebot sobre "Educación en Colombia en el Siglo XX", en Boletín No. 249, abril 1972.

GONZALEZ, Jorge Iván Tasa de rendimiento de la Educación, productividad y nivel de pobreza en la pequeña industria, en UNIANDES, Revista Desarrollo y Sociedad, No.7, págs. 147-162.

KUGLER, B. El papel de la Educación secundaria en el mercado de trabajo, en Revista Colombiana de Educación, No.10, 1982, CIUP.

SENA Educación, formación profesional y empleo. Bogotá, mayo de 1984.

SENALDE Transición demográfica y oferta de fuerza de trabajo en Colombia. Imprenta Nacional. Bogotá, 1986, Tomos I y II. 